\title{
Cleaning spectroscopic samples of stars in nearby dwarf galaxies
}

\section{The use of the nIR Mg I line to weed out Milky Way contaminants}

\author{
G. Battaglia ${ }^{1,2}$ and E. Starkenburg ${ }^{3}$ \\ ${ }^{1}$ European Organization for Astronomical Research in the Southern Hemisphere, K. Schwarzschild-Str. 2, 85748 Garching, Germany \\ 2 INAF - Osservatorio Astronomico di Bologna, via Ranzani 1, 40127 Bologna, Italy \\ e-mail: gbattaglia@oabo.inaf.it \\ 3 Kapteyn Astronomical Institute, University of Groningen, PO Box 800, 9700 AV Groningen, The Netherlands \\ e-mail: else@astro.rug.nl
}

Received 24 June 2011 / Accepted 16 January 2012

\begin{abstract}
Dwarf galaxies provide insight into the processes of star formation and chemical enrichment at the low end of the galaxy mass function, as well as into the clustering of dark matter on small scales. In studies of Local Group dwarf galaxies, spectroscopic samples of individual stars are used to derive the internal kinematics and abundance properties of these galaxies. It is therefore important to clean these samples from Milky Way stars, which are not related to the dwarf galaxy, since they can contaminate analysis of the properties of these objects. Here we introduce a new diagnostic for separating Milky Way contaminant stars, which mainly consist of dwarf stars, and red giant branch stars targeted in dwarf galaxies. As discriminator we use the trends in the equivalent width of the nIR $\mathrm{Mg}$ I line at $8806.8 \AA$ as a function of the equivalent width of Ca II triplet lines. This method is particularly useful for works dealing with multi-object, intermediate-resolution spectroscopy focusing in the region of the nIR Ca II triplet. We use synthetic spectra to explore how the equivalent width of these lines changes for stars with different properties (gravity, effective temperature, metallicity) and find that a distinction among giants above the horizontal branch and dwarfs can be made with this method at $[\mathrm{Fe} / \mathrm{H}]>-2$ dex. For $-2 \leq[\mathrm{Fe} / \mathrm{H}] \leq-1$, this method is also valid for distinguishing dwarfs and giants down to approximately one magnitude below the horizontal branch. Using a foreground model we make predictions on the use of this new discrimination method for nearby dwarf spheroidal galaxies, including the ultra-faints. We subsequently use VLT/FLAMES data for the Sextans, Sculptor, and Fornax dwarf spheroidal galaxies to verify the predicted theoretical trends.
\end{abstract}

Key words. stars: abundances - galaxies: evolution - galaxies: dwarf - Local Group - galaxies: stellar content - Galaxy: formation

\section{Introduction}

There is much to be learned from the galaxies within the Local Group because they can be studied in better detail than any other system, on a star-by-star basis. Within the Milky Way (MW) halo itself, we find satellite galaxies that span a range over ten magnitudes in brightness. The great majority of the MW satellites belong to the class of early type dwarf galaxies, which are the smallest and least luminous galaxies known to date. These include the ultra faint dwarfs (UFDs), which were relatively recently discovered with the Sloan Digital Sky Survey (SDSS). Several future surveys, such as Pan-STARRS (Kaiser et al. 2002) and Skymapper (Keller et al. 2007), are expected to further increase the number of detected dwarf galaxies around the MW, towards lower surface brightnesses.

Much effort has been devoted to the study of these small early type galaxies (see Tolstoy et al. 2009, for a review) since they provide precious insight into the processes that drive galaxy evolution at the low end of the galaxy mass function, as well as into the clustering of dark matter on small scales (e.g. Gilmore et al. 2007). Since stars in these galaxies are resolved, the internal properties for the galaxies are derived from photometric and spectroscopic studies of individual objects. This requires application of methods to discard foreground/background stars,

* Based on FLAMES observations collected at the ESO, proposals 171.B-0588, 076.B-0391, 079.B-0435. which are not bound to the dwarf galaxy, but that are instead within the MW itself (hereafter "contaminants" or "interlopers") and would influence the properties derived for the dwarf galaxy.

General methods of excluding obvious interlopers include using color-magnitude diagrams (CMDs) to select a region in magnitude and color where one would expect member stars in a certain stellar evolutionary stage to lie, given the distance to the object. For most of the studies of Local Group early type dwarf galaxies, a photometric selection box is placed on the red giant branch (RGB), the most luminous feature in the CMD of ancient/intermediate age stellar populations. However, such a selection box will also contain contaminants, which consist predominantly of much intrinsically fainter, but close-by main sequence dwarf stars from the MW thin or thick disk. To distinguish the contaminant dwarf stars from the RGB stars members to the dwarf galaxy additional discriminators need to be used.

Spectroscopic studies can rely on another selection criterion to weed out foreground contamination, by using the line-of-sight (1.o.s.) velocity of the individual stars as compared to the systemic velocity of the dwarf galaxy. This is commonly done in a statistical sense, either using sigma-clipping procedures (which imply a negligible probability to find a member beyond a certain velocity threshold) or maximum likelihood analyses, with more detailed implementations modeling both the expected population of dwarf galaxy stars and MW stars (e.g. Battaglia et al. 2008a; Walker et al. 2009b; Martinez et al. 2011). More properties than 
1.o.s. velocities can be included in the analysis, e.g. distance of the star from the galaxy center (e.g. Battaglia et al. 2008a) and information on spectral indices (Walker et al. 2009b, for the $\mathrm{Mg}$ index around $\lambda 5170 \AA$ ) or metallicity (Martinez et al. 2011) of the star. The most sophisticated of these implementations from a statistical point of view are those of Walker et al. (2009b) and Martinez et al. (2011).

However, even the most efficient of these methods cannot by construction select out MW contaminants with similar properties (colors, apparent magnitudes, l.o.s. velocities, etc.) to the member stars to the observed galaxy. Depending on the type of stars surveyed, the systemic velocity of the galaxy and its position on the sky (most importantly Galactic latitude), contaminant stars satisfying all these requirements might still be numerous or at least statistically significant in number. Furthermore, a selection criterion based on position and velocity (and/or metallicity) needs assumptions about the underlying distribution and can therefore also bias the final analysis. For example, it is unclear whether features in the outer parts could be mistaken for contaminants: if looking for tidally disrupted stars, one expects to find them in the very outer parts of the dwarf and at velocities that are rather discrepant from the systemic (e.g. Muñoz et al. 2006, for an observational study of tidal disruption in Carina). In statistical procedures those stars would most likely not be considered as members. Similarly, stars in the outer "shell" of the Fornax dSph (Coleman et al. 2005), arguably the remnant of an accretion event, would most likely be rejected because they are well outside the limiting radius of the dwarf, as inferred by its surface brightness profile. Also, stars with very low and high metallicity with respect to the rest of the population, could be rejected as non-members, while true members with deviating metallicities could offer important insight into the earliest phases of chemical enrichment and into the capability of the dwarf to retain gas and/or metals, respectively.

These examples illustrate the benefits of also having methods that weed out contaminants on the basis of sensitivity to gravity, since the stars targeted in external galaxies are RGB stars, and the foreground consists mostly of dwarf stars from the thin or thick disk. Such a discriminator can be used separately or combined with other (statistical) methods to increase the efficiency of the elimination of interlopers.

Photometric techniques are present in the literature that allow a distinction between dwarf and giant stars by constructing indices sensitive to gravity and luminosity from combinations of particular filters. For example, the DDO51 filter is centered on the $\mathrm{Mg} \mathrm{b} / \mathrm{MgH}$ feature near $5170 \AA$, which is strong in late-type dwarfs and weak in giants (e.g. Morrison et al. 2001). Several works have shown the effectiveness of separating giant and dwarf stars using a combination of Washington and DDO filters. Muñoz et al. (2006) photometrically preselected their spectroscopic targets using the M51 filter and find probable members of the Carina $\mathrm{dSph}$ out to very large distances from its center, where genuine members would have otherwise been buried in the foreground. Faria et al. (2007) used Strömgren photometry on the Draco dSph, showing that the Strömgren $c_{1}$ index, which is sensitive to luminosity, can be used together with the $(b-y)_{0}$ color to separate stars that are in various evolutionary stages. While photometric techniques provide broad information on the star formation history of the dwarf galaxy and in some cases on the metallicity distribution of its stars (e.g. Faria et al. 2007), information on the l.o.s. velocity of the individual stars appears crucial to assess membership in some cases even when using Washington photometry (e.g. Morrison et al. 2001).
Spectroscopic studies also exist of early type Local Group dwarf galaxies that have used features in the spectra of the stars to distinguish between giants and dwarfs. Spinrad \& Taylor (1971) and Schiavon et al. (1997) showed that the Na I doublet of absorption lines at $8183,8195 \AA$ is strongly dependent on gravity and temperature. Several studies have adopted the equivalent width (EW) of the $\mathrm{Na}$ I doublet as a diagnostic to differentiate dwarf and giant stars, with the dwarf stars displaying much larger EWs than giant stars (see Gilbert et al. 2006; Simon \& Geha 2007; Martin et al. 2007, on the halo of M 31 for the former, and on UFDs for the last two works). Gilbert et al. (2006) show that this diagnostic offers clear differentiation at colors $(V-I)>2$, while there appears to be significant overlap in the distribution of $\mathrm{Na}$ I doublet EW of dwarf and giant stars for bluer colors.

The adoption of one method, or more, to assess membership depends on the particular scientific problem, galaxy, and dataset under consideration, and the fraction of contaminants that can be tolerated so as not to significantly affect the results. In some cases, the presence of interlopers has a dramatic impact on the conclusions; for example, mass estimates derived from measurements of the l.o.s. velocity dispersion of samples of individual stars in Local Group early type dwarf galaxies have been used to discuss the possible existence of "universal" mass value (e.g. Strigari et al. 2008; Walker et al. 2009a). In their study of UFDs, Simon \& Geha (2007) have adopted a wealth of membership indicators, such as spatial position of the star, location on the CMD, EW of the Na I doublet, l.o.s. velocity, and metallicity, and find an internal velocity dispersion of $5.1 \pm 0.9 \mathrm{~km} \mathrm{~s}^{-1}$ for the Hercules dSph. By removing MW stars using Strömgren photometry, Adén et al. (2009a) find a lower velocity dispersion in their cleaned sample, $3.7 \pm 0.9 \mathrm{~km} \mathrm{~s}^{-1}$. In this particular example, the derived mass of Adén et al. (2009b) from this new velocity dispersion places Hercules well below the "common mass scale" for MW early type dwarf galaxies (Strigari et al. 2008, and references therein).

Another instructive example is the case of Willman 1, whose apparent spread in the metal abundance distribution was used in favor of its classification as a very low-luminosity dwarf galaxy (Martin et al. 2007). Subsequent studies based on highresolution spectroscopic observations have revealed that a large percentage of stars classified as members on the basis of their position on the CMD, velocity, and EW of the Na I doublet by Martin et al. (2007) are either foreground dwarfs or halo giants, renewing the possibility that Willman 1 could be a disrupted metal-poor globular cluster.

The examples above show the importance of careful interloper removal by using as many membership indicators as possible. This is particularly important when the galaxies studied become fainter and consist of fewer stars, and for studies that explore the outer regions of dwarf galaxies, where the expected ratio of members/interlopers is high.

In this paper we present an additional discriminator to separate dwarf and giant stars, i.e. the $\mathrm{EW}$ of the $\mathrm{Mg}$ I line at $8806.8 \AA$ used in combination with the sum of the EW of the two strongest $\mathrm{Ca}$ II triplet $(\mathrm{CaT})$ lines $(\Sigma \mathrm{W})$, at $\lambda=8542.1$ and $8662.1 \AA$ (see Fig. 1). This method was empirically introduced by Battaglia et al. (2011, hereafter B11) as a criterion for discriminating RGB stars belonging to the Sextans dSph from the MW contaminant stars. Using a data-set with a limited extent in metallicity, the authors noted that the great majority of the stars with l.o.s. velocities very close to the systemic of Sextans (i.e. highly likely member RGB stars) clustered around 


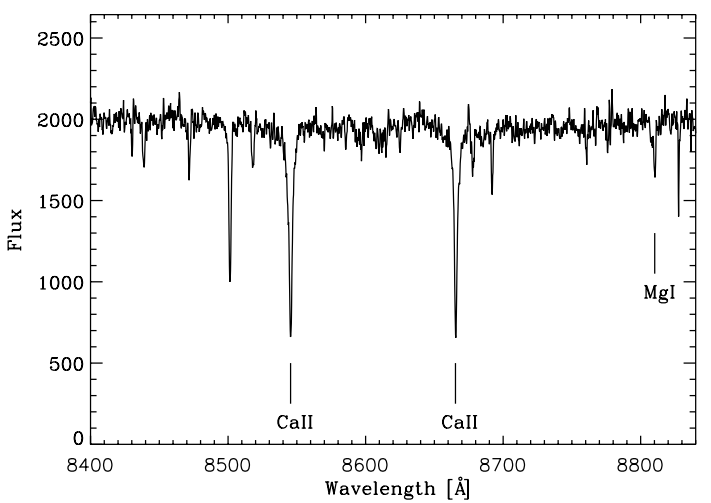

Fig. 1. VLT/FLAMES spectrum from DART observations of a star along the l.o.s. to the Sculptor dSph (heliocentric velocity $=122.7 \mathrm{~km} \mathrm{~s}^{-1}$ and signal-to-noise ratio $\left.\mathrm{S} / \mathrm{N} / \AA=64\right)$. The lines used in this work, i.e. the two strongest $\mathrm{CaT}$ lines and one $\mathrm{Mg}$ I line, are marked.

low values of the $\mathrm{Mg} \mathrm{I} \mathrm{EW}$, while stars with velocities more than $4 \sigma$ away from the systemic (i.e. highly likely non-members) clustered at higher values, $>0.5 \AA$. In this paper we extend the empirical work of B11, by using synthetic spectra to explore the validity of the method over a range of metallicities, gravities, and $\Sigma \mathrm{W}$, and demonstrating the effectiveness of using the $\mathrm{Mg}$ I line in combination to the CaT lines in removing MW contaminants.

This method is a convenient choice, because $\mathrm{MgI}$ and CaT lines are close in wavelength space and relatively broad, which means they will typically both be present and measurable in intermediate-resolution studies of this wavelength regime. They are located in the red part of the spectrum, where the target RGB stars are brightest, with optimization of the exposure time. Furthermore, the wavelength region including $\mathrm{CaT}$ lines has become increasingly used in intermediate-resolution spectroscopic studies of Local Group early type dwarf galaxies because CaT lines are both very suitable for radial velocity determinations and offer the possibility of deriving estimates of $[\mathrm{Fe} / \mathrm{H}]$ accurate to $\pm 0.15-0.2$ dex over a wide metallicity range (Starkenburg et al. 2010) for large numbers of individual stars and much lower observing times than in high-resolution studies (typically an $\mathrm{S} / \mathrm{N} \sim 10$ is required). The method can be applied to a wealth of galaxies observed in this wavelength regime. In the ESO archive a large body of data already exists at intermediate resolution in the only set-up that includes the CaT, which covers the region between 8206 and $9400 \AA$. This is by far the most frequently used set-up for the FLAMES instrument. The Na I doublet is just outside this wavelength range. The current archive includes observations of Fornax, Sculptor, Sextans, Carina, Leo II, Leo IV, Hercules, Boo I, IC 1613, Sag, and Segue 1 in this wavelength range, by various groups. The $\mathrm{CaT}$ region has also been targeted by studies using other telescopes than VLT, such as the work of Koch et al. (2007) on Leo I. Furthermore, the future large GaiaESO Survey (GES) will target the wavelength region covering the nIR CaT and Mg I line (V. Hill, GES Team, priv. comm.). Several existing and future datasets could therefore benefit from this discriminator.

The paper is structured as follows. In Sect. 2 we use synthetic spectra to explore the trends between the EW of $\mathrm{Mg}$ I line and CaT lines for stars of different metallicities and gravities and to derive a relation that allows us to distinguish between giant and dwarf stars on the basis of these lines. We then comment on the expected success rate of this method along the l.o.s. to different MW satellites in Sect. 3. In Sect. 4 we use data for the Sextans,

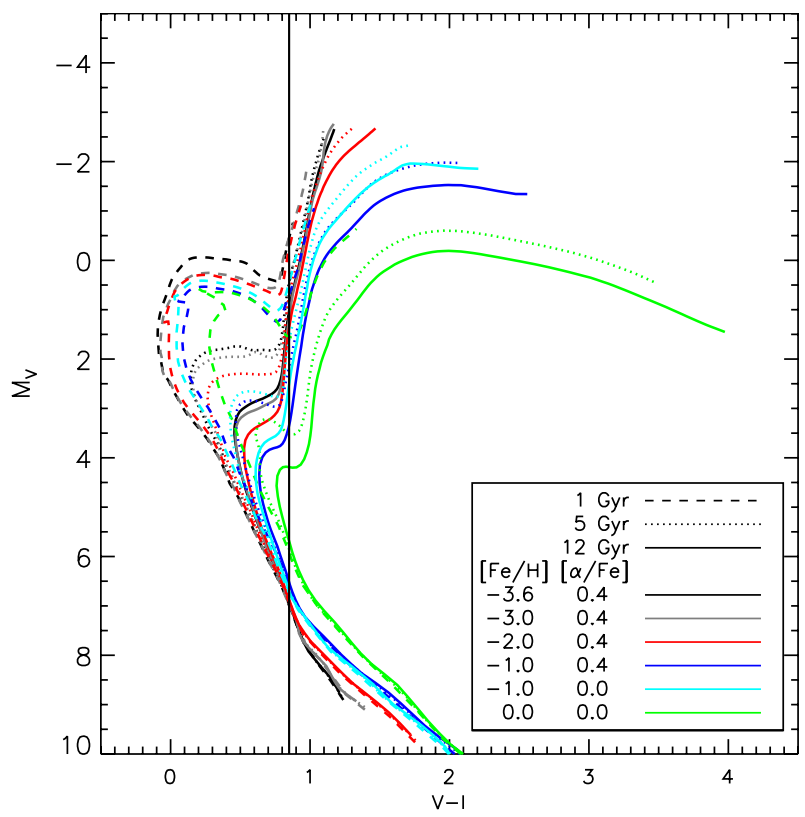

Fig. 2. Color-magnitude diagram using Yonsei-Yale isochrones for $1 \mathrm{Gyr}$ (dashed lines), 5 Gyr (dotted lines), and $12 \mathrm{Gyr}$ (full lines), and metallicities $[\mathrm{Fe} / \mathrm{H}]=-3.6$ (lowest value possible within the Yonsei-Yale set in black), $[\mathrm{Fe} / \mathrm{H}]=-3.0$ (gray), $[\mathrm{Fe} / \mathrm{H}]=-2.0$ (red), $[\mathrm{Fe} / \mathrm{H}]=-1.0$ (blue), $[\mathrm{Fe} / \mathrm{H}]=0.0$ (green). $[\alpha / \mathrm{Fe}]$ is set to +0.4 for all isochrones except for $[\mathrm{Fe} / \mathrm{H}]=0.0$, which have $[\alpha / \mathrm{Fe}]=0.0$ and an extra set of isochrones plotted for $[\mathrm{Fe} / \mathrm{H}]=-1$ and $[\alpha / \mathrm{Fe}]=0.0$ (cyan). The vertical line roughly indicates the onset of the RGB.

Sculptor, and Fornax dSphs from the Dwarf Abundances and Radial velocities Team (DART, Tolstoy et al. 2006), to explore the behavior of the lines empirically for galaxies with different characteristics, compare the results to the models, and show the agreement between models and observations. Finally we apply the method to the DART dataset in Sect. 4.1 and present our conclusions in Sect. 5.

\section{The lines and their sensitivity}

To investigate the behavior of the nIR CaT and $\mathrm{Mg}$ I absorption lines, we first have to define the boundaries of the physical parameter space we are working in. In practice, we do this using isochrones covering possible ranges of $[\mathrm{Fe} / \mathrm{H}]$ and ages as covered by the stars in Local Group dwarf galaxies and MW foreground in order to select the appropriate regions in $\log (g)-T_{\text {eff }}$ space (see Fig. 2). The isochrones we use are Yonsei-Yale isochrones (e.g. Yi et al. 2001; Demarque et al. 2004) for 1, 5, and $12 \mathrm{Gyr}$, covering the wide range $-3.6 \leq[\mathrm{Fe} / \mathrm{H}] \leq+0.0$. To represent general trends within the MW stars, the alpha enhancements chosen are +0.4 for $[\mathrm{Fe} / \mathrm{H}] \leq-1$ dex and +0.0 for the solar $[\mathrm{Fe} / \mathrm{H}]$. However, since spectroscopic observations of stars in the Sculptor (e.g. Tolstoy et al. 2009), Fornax (Letarte et al. 2010), and Carina (Koch et al. 2008) dSphs show $[\alpha / \mathrm{Fe}]$ around solar values already at $[\mathrm{Fe} / \mathrm{H}] \sim-1 \mathrm{dex}$, for completeness we add an isochrone set of $[\alpha / \mathrm{Fe}]=0.0 \mathrm{dex}$ and $[\mathrm{Fe} / \mathrm{H}]=-1 \mathrm{dex}$.

Because the dwarf galaxies currently studied in large spectroscopic surveys (e.g. Walker et al. 2006, 2009b; Simon \& Geha 2007; Adén et al. 2009a; Kirby et al. 2010) are distant objects, typically only the giants above the horizontal branch (HB) will be bright enough to be followed up with spectroscopy; however, in those systems that have sparsely populated RGBs, the observations often need to be pushed to fainter magnitudes, 
therefore below the HB (e.g. Martin et al. 2007; Simon \& Geha 2007). The isochrones plotted in Fig. 2 show that giant stars of any metallicity or age above the HB (which will have $0<$ $M_{V}<+1$ typically Rich et al. 2005; Catelan \& Cortés 2008), and most of those below the HB, will not be found at bluer colors than $V-I \sim 0.85$, corresponding to stars hotter than $\sim 5250 \mathrm{~K}$ (Ramírez \& Meléndez 2005). In our following analysis, aiming at disentangling the populations of giant and dwarf stars which would be preselected photometrically, we therefore will not take temperatures $>5250 \mathrm{~K}$ into account. This temperature selection cuts out an important fraction of the hot, halo and thick disk stars in the foreground contamination. Figure 2 also shows that at the lowest metallicity end the isochrones become less sensitive to metallicity and thus we expect no significant changes if even lower metallicities $([\mathrm{Fe} / \mathrm{H}]=-4$ dex and below $)$ are considered in the analysis.

On the other end of the temperature scale, we place a cutoff at $V-I=2.1$, corresponding to a temperature of $3600 \mathrm{~K}$. Although in theory very metal-rich giants stars can reach even cooler temperatures, these are rare in dwarf galaxies because most dwarf galaxies have low metallicity. Indeed, following this consideration, most photometric boxes that have been used for preselecting spectroscopic targets are well within this range. With this cut, the only population of giant stars completely excluded from the analysis is the one of super-solar metallicity and with an age of $12 \mathrm{Gyr}$, unexpected in such systems.

Since we address in this work the validity of the use of the $\mathrm{Mg}$ I and CaT lines to distinguish RGB stars in Local Group dwarf galaxies from MW contaminants, we use in the analysis synthetic spectra with physical properties $\left(\log (g), T_{\text {eff }}\right.$, etc.) covering the range expected for both populations of stars within the color range discussed above. We calculated a grid of synthetic spectra for a wide range of metallicities, temperatures, and gravity using (OS)MARCS model atmospheres (e.g. Gustafsson et al. 2008; Plez 2008) and the Turbospectrum program (Alvarez \& Plez 1998), updated consistently with the Gustafsson et al. (2008) MARCS release. In agreement with the trends followed by most of the stars in the MW (e.g. Venn et al. 2004), we adopt MARCS models with "standard" chemical composition, i.e. the values chosen for the alpha elements (taken as $\mathrm{O}, \mathrm{Ne}$, $\mathrm{Mg}, \mathrm{Si}, \mathrm{S}, \mathrm{Ar}, \mathrm{Ca}$, and $\mathrm{Ti}$ ) relative to $\mathrm{Fe}$ for the models are $[\alpha / \mathrm{Fe}]=+0.4$ at the metallicities below solar, linearly decreasing towards $[\alpha / \mathrm{Fe}]=0.0$ at solar $[\mathrm{Fe} / \mathrm{H}]$. The microturbulent velocities used in our models are $1-2 \mathrm{~km} \mathrm{~s}^{-1}$, in agreement with the observed range (e.g. Barklem et al. 2005).

To allow a comparison of the data with the synthetic spectra we define regions in $T_{\text {eff }}-\log (g)$ space where the giants above the $\mathrm{HB}$, (sub)giants below the $\mathrm{HB}$ and main-sequence branches would lie (see upper panels of Fig. 3). For "classical" dSphs, which have a well-populated RGB, spectroscopic samples mostly target RGB stars above the HB, therefore the member stars we are looking for will all fall within the "giant" boxes (green boxes in Fig. 3). For those UFDs for which observations must be pushed below the HB, members will also fall within the "sub-giant" boxes. For all the models that fall within either one of the selection boxes we measure the EW of the $\mathrm{MgI}$ line and of the two strongest CaT lines in the corresponding synthetic spectrum. We measure the EW of the CaT lines using a Gaussian fit to the individual lines, corrected by a factor 1.1 to include the non-Gaussian wings. This factor is determined by comparing the EW from the Gaussian fit to the one from the integrated line measure (for more details see Battaglia et al. 2008b; and Starkenburg et al. 2010). We also correct the EWs of the CaT lines for non-LTE effects using the equations given in Starkenburg et al. (2010). These corrections are mainly important for the very low-metallicity regime. The $\mathrm{Mg}$ Iline is measured using a simple integration of the area under the continuum of $6 \AA$ around the line, as also done for the data (see Sect. 4).

In the bottom panel of Fig. 3, we show the sum of the EW for the two strongest CaT lines $\left(\Sigma \mathrm{W}_{\mathrm{CaT}}\right)$ versus the $\mathrm{EW}$ of the $\mathrm{Mg}$ I line $\left(\mathrm{EW}_{\mathrm{Mg}}\right)$. For $[\mathrm{Fe} / \mathrm{H}]=-2$ and -1 dex the dwarf stars can be separated from the giants and subgiants. At solar metallicity, the distinction remains clear between the dwarfs and the giants, but not for the sub-giants. We note though that, given the metallicity-luminosity relation (see Kirby et al. 2010, for early type dwarf galaxies in the Local Group), stars with metallicities close to solar are not expected in UFDs, which are the systems for which one often needs to target stars below the HB due to their sparsely populated RGB. The figure also shows that the distinction among the different classes of objects would not be possible if one were to collapse all data onto one axis (i.e. would use just one of the lines): it is the combination of these lines that makes the distinction between the giants and dwarfs possible in most cases. In the extremely low-metallicity regime (i.e., $[\mathrm{Fe} / \mathrm{H}]=-3)$, giants, subgiants and dwarfs appear to occupy the same region in the $\mathrm{EW}_{\mathrm{Mg}}, \Sigma \mathrm{W}_{\mathrm{CaT}}$ plane. However, this is not a concern because in Sect. 3 - where we discuss the expected contamination for different objects - we show that interloper stars with such low metallicities are very rare.

A distinction line between giants and dwarfs is overplotted in all panels and is

$$
\mathrm{EW}_{\mathrm{Mg}}(\mathrm{m} \AA)= \begin{cases}300 & \text { if } \Sigma \mathrm{W}_{\mathrm{CaT}} \leq 3750 \mathrm{~m} \AA \\ 0.26 \times \Sigma \mathrm{W}_{\mathrm{CaT}}-670.6 & \text { if } \Sigma \mathrm{W}_{\mathrm{CaT}}>3750 \mathrm{~m} \AA .\end{cases}
$$

As we discuss in Sect. 3, the fraction and type of contaminant stars, and therefore their location and importance on the $\mathrm{EW}_{\mathrm{Mg}}$ vs. $\Sigma W_{\mathrm{CaT}}$ plane, will change according to the specifics of the dwarf galaxy (position on the sky, systemic velocity, distance). This line therefore provides a criterion for keeping as many of the member stars of the dwarf galaxy in, and throwing away as many contaminants as one can.

\section{The foreground contamination}

\subsection{Fornax, Sculptor, and Sextans}

As a test case of different mixtures of MW interlopers we look at the expected contamination along three different l.o.s., specifically to the Sextans, Sculptor and Fornax dSphs.

To study the likely foreground contamination towards these objects, we use the Besançon model (Robin et al. 2003). All interloper stars were selected within a $4 \mathrm{deg}^{2}$ solid angle around these galaxies, at a distance between 0 and $200 \mathrm{kpc}$. As in Sect. 2, we restrict the analysis to the color range $0.85<V-I<2.1$. We also make a selection in apparent magnitude, which would include giant stars above the HB at the distance of these "classical" MW dSphs. For this we use the distance moduli from the literature and information on the HB magnitudes (see Table 1), and we adopt an absolute magnitude in $I$-band $M_{I}=-4$ for the tip of the RGB. This range in apparent magnitude will also be populated by a range of MW contaminants - mainly main sequence dwarf stars - with the appropriate combination of absolute magnitude and distance from the Sun.

The leftmost panels of Fig. 4 show the foreground sample in the direction of these three dwarf galaxies (in all these panels the 
G. Battaglia and E. Starkenburg: The nIR Mg I line to weed out Milky Way contaminants

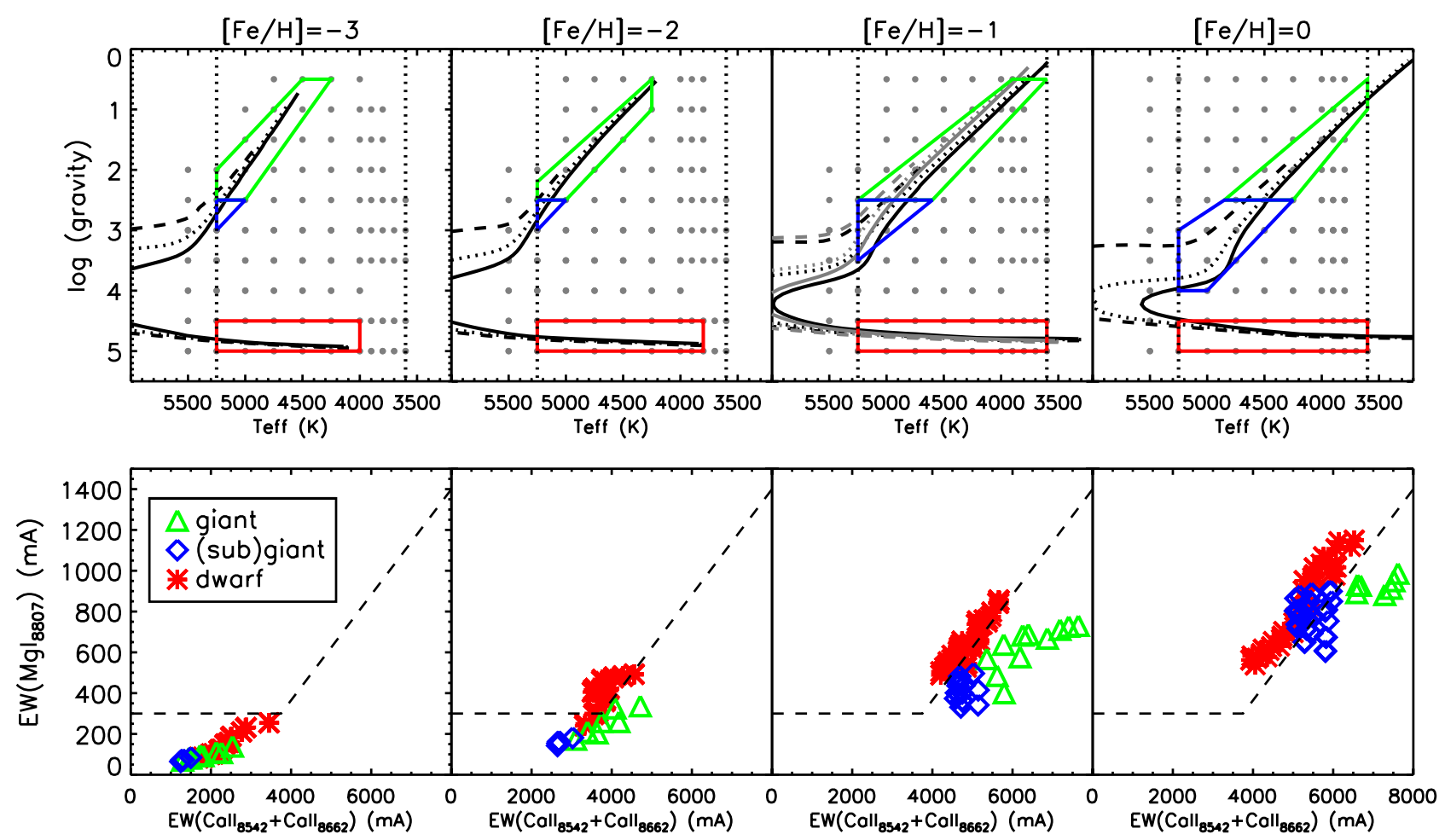

Fig. 3. Upper panels: Yonsei-Yale isochrones for $1 \mathrm{Gyr}$ (dashed lines), $5 \mathrm{Gyr}$ (dotted lines), and $12 \mathrm{Gyr}$ (full lines) for one particular metallicity. For $[\mathrm{Fe} / \mathrm{H}]=-3$ and $[\mathrm{Fe} / \mathrm{H}]=-2,[\alpha / \mathrm{Fe}]=0.4$, for $[\mathrm{Fe} / \mathrm{H}]=0.0,[\alpha / \mathrm{Fe}]=0.0$ and for $[\mathrm{Fe} / \mathrm{H}]=-1$ both alpha enhancements are given $([\alpha / \mathrm{Fe}]=0.0$ in gray). All values for which the synthetic spectra are calculated are covered with a gray dot. Overplotted are regions where the giants above the HB (green), (sub)giants below the HB (blue) and main-sequence (red) branches would lie in $T_{\text {eff }}-\log (g)$ space, if the temperature restrictions from photometry are taken into account (vertical dotted lines). Lower panels: EWs for the two strongest CaT line versus the MgI 8806.8 line for models that fall within the boxes of the panel above. Giants above the HB are shown as green triangles, (sub)giants below the HB as blue diamonds and dwarfs as red asterisks. Overplotted is a distinction line for dwarfs and giants (except for the extremely low-metallicity case), and is the same in all panels.

Table 1. Properties of the foreground contamination towards the MW satellites.

\begin{tabular}{|c|c|c|c|c|c|c|c|c|c|c|c|c|}
\hline Galaxy & $\begin{array}{c}l \\
\left({ }^{\circ}\right) \\
\end{array}$ & $\begin{array}{c}b \\
\left(^{\circ}\right) \\
\end{array}$ & $\mathrm{dm}$ & $V_{\mathrm{HB}}$ & $\begin{array}{c}v_{\text {sys }} \\
{\left[\mathrm{km} \mathrm{s}^{-1}\right]}\end{array}$ & $\begin{array}{c}\sigma \\
{\left[\mathrm{km} \mathrm{s}^{-1}\right]}\end{array}$ & $\begin{array}{c}N_{\text {cont }} \\
/ 4 \mathrm{deg}^{2} \\
\end{array}$ & $\begin{array}{c}\%\left(N_{\text {cont }}\right) \\
\text { vel. crit. }(3 \sigma) \\
\end{array}$ & $\begin{array}{l}\%\left(N_{\text {cont }}\right) \\
\text { line crit. }\end{array}$ & $\begin{array}{c}\%\left(N_{\text {cont }}\right) \\
\text { both }(3 \sigma)\end{array}$ & $\begin{array}{c}\%\left(N_{\text {cont }}\right) \\
\text { both }(4 \sigma)\end{array}$ & Ref. \\
\hline Fornax & 237.1 & -65.7 & 20.7 & 21.29 & 54.1 & 11.4 & 2309 & $44 \%$ & $17 \%$ & $6 \%$ & $8 \%$ & $1,2,3$ \\
\hline Sculptor & 287.5 & -83.2 & 19.54 & 20.13 & 110.6 & 10.1 & 1872 & $4 \%$ & $16 \%$ & $2 \%$ & $3 \%$ & $1,2,4$ \\
\hline Sextans & 243.5 & 42.3 & 19.67 & 20.35 & 226.0 & 8.4 & 3687 & $0.9 \%$ & $12 \%$ & $0.5 \%$ & $0.6 \%$ & $1,2,5$ \\
\hline Canes Venatici II & 74.3 & 79.8 & 20.9 & & -128.9 & 4.6 & 2891 & $2 \%$ & $14 \%$ & $0.9 \%$ & $1 \%$ & 6,7 \\
\hline Canes Venatici I & 113.6 & 82.7 & 21.75 & 22.4 & 30.9 & 7.6 & 2283 & $21 \%$ & $19 \%$ & $3 \%$ & $5 \%$ & $8,9,7$ \\
\hline Carina & 260.1 & -22.2 & 20.03 & 20.50 & 223.9 & 7.5 & 18000 & $0.7 \%$ & $16 \%$ & $0.5 \%$ & $0.7 \%$ & $1,2,10$ \\
\hline Coma Berer & 241.9 & 83.6 & 18.2 & & 98.1 & 4.6 & 1738 & $1 \%$ & $11 \%$ & $0.5 \%$ & $0.7 \%$ & $6,11,7$ \\
\hline Draco & 86.4 & 34.7 & 19.58 & 20.07 & -293.0 & 9.1 & 6329 & $0.5 \%$ & $15 \%$ & $0.4 \%$ & $0.5 \%$ & $1,2,12$ \\
\hline Hercules & 28.7 & 36.9 & 20.7 & 21.17 & 45.0 & 5.1 & 13368 & $13 \%$ & $22 \%$ & $3 \%$ & $4 \%$ & $6,13,7$ \\
\hline Leo I & 226.0 & 49.1 & 21.99 & 22.30 & 286.0 & 9.2 & 2617 & $1 \%$ & $16 \%$ & $0.3 \%$ & $0.5 \%$ & $1,2,12$ \\
\hline Leo II & 220.2 & 67.2 & 21.63 & 22.30 & 76.0 & 6.6 & 2248 & $12 \%$ & $19 \%$ & $3 \%$ & $4 \%$ & $1,2,12$ \\
\hline Leo IV & 265.4 & 56.5 & 21.0 & & 132.2 & 7.6 & 3930 & $6 \%$ & $13 \%$ & $2 \%$ & $2 \%$ & 6,7 \\
\hline Segue I & 220.5 & 50.4 & 16.8 & & 208.5 & 3.7 & 1637 & $0.2 \%$ & $9 \%$ & $0.1 \%$ & $0.2 \%$ & 6,14 \\
\hline Ursa Major I & 152.5 & 37.4 & 20.13 & 20.5 & -55.3 & 7.6 & 4096 & $24 \%$ & $11 \%$ & $3 \%$ & $4 \%$ & 7,9 \\
\hline Ursa Major II & 159.4 & 54.4 & 17.5 & & -116.5 & 6.7 & 1868 & $2 \%$ & $10 \%$ & $0.9 \%$ & $1 \%$ & 15,7 \\
\hline Ursa Minor & 105.0 & 44.8 & 19.11 & 19.80 & -248 & 9.5 & 3449 & $0.8 \%$ & $14 \%$ & $0.6 \%$ & $0.8 \%$ & $1,2,12$ \\
\hline Willman 1 & 158.6 & 56.8 & 17.9 & & -12.3 & 4.3 & 2026 & $32 \%$ & $8 \%$ & $1 \%$ & $2 \%$ & 9 \\
\hline
\end{tabular}

Notes. $N_{\text {cont }} / 4 \mathrm{deg}^{2}$ is the number of contaminants contained within a solid angle of $4 \mathrm{deg}^{2}$ along the l.o.s. to the galaxy. The last 4 columns list the fraction of contaminants retained when using only the velocity criterion, the line criterion, when applying both, and applying both relaxing the velocity selection to $4-\sigma$.

References. (1) Mateo (1998); (2) Irwin \& Hatzidimitriou (1995); (3) Battaglia et al. (2006); (4) Battaglia et al. (2008b); (5) B11; (6) Belokurov et al. (2007); (7) Simon \& Geha (2007); (8) Zucker et al. (2006b); (9) Martin et al. (2007); (10) Koch et al. (2006); (11) Belokurov et al. (2007); (12) Walker et al. (2009b); (13) Adén et al. (2009a); (14) Simon et al. (2011); (15) Zucker et al. (2006a). 


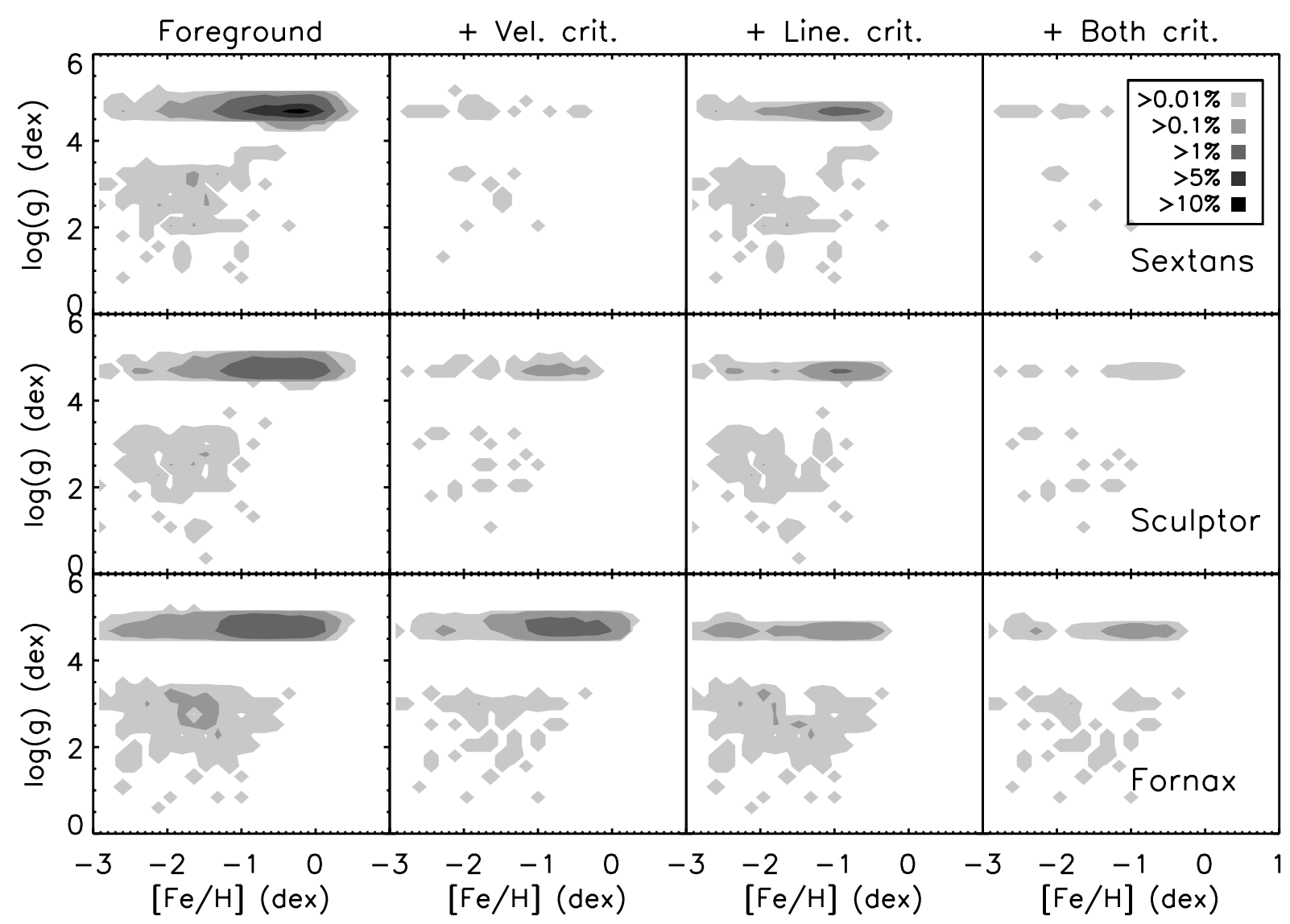

Fig. 4. Left panels: $[\mathrm{Fe} / \mathrm{H}]$ and $\log (g)$ for all foreground stars drawn from the Besançon model towards the direction of the dwarf spheroidal galaxies Sextans (top row), Sculptor (middle row), and Fornax (bottom row). Contours are shown on a 25 by 25 grid. The rest of the panels show the distribution of foreground stars left after applying a velocity criterion of $3 \sigma$ around the systemic velocity of the galaxy, a line criterion as described in the text on the basis of the EWs of the CaT and Mg I lines, and both criteria combined. Percentage levels are the same in all panels and always relative to the total number of foreground stars towards that galaxy.

contour levels are not linear), while the number of contaminants is given in Table 1. It is clear that the type of stars within the foreground sample will depend on the direction in the sky. Sculptor, as the galaxy at highest latitude, has the least foreground in general, but a relatively high fraction of its foreground are giant stars, which are difficult to weed out with our method. Although all these galaxies are at high(ish) latitudes, there is still a very significant contamination from the disk in all cases, in particular from the thick disk. The thin and thick disks contribute almost entirely dwarf stars $(\log (g)>4.3)$, while the fraction drops to about $50 \%$ for the contribution of dwarf stars from the halo. As previously discussed, the figure also shows that the great majority $(>90 \%)$ of the contaminants are found at $[\mathrm{Fe} / \mathrm{H}]>-2$ dex, where the distinction between giants and dwarfs is easier. The stars with $[\mathrm{Fe} / \mathrm{H}]<-2$ dex mostly belong to the stellar halo, which is the least dominant population in all of these cases.

In previous work, the member stars of the dwarf galaxy were selected out from the contaminants on the basis of their l.o.s. velocities. The stars were, for example, required to have a l.o.s. velocity within $3 \sigma$ from the systemic velocity of the galaxy. For the Fornax dwarf galaxy $2.5 \sigma$ was used, because its systemic velocity is closer to the velocity of the Galactic disk (Battaglia et al. 2006). Using the simulated catalogs of MW contaminants we find that, in all cases, as expected, some foreground stars will remain that have similar velocities to the dwarf galaxy stars, as is shown in the second column in Fig. 4. The fraction, though, changes significantly from galaxy to galaxy. For Sculptor and
Sextans the fraction of interlopers retained by the velocity criterion is low, as listed in Table 1 , but the cleaning by velocity is much less effective in the Fornax dwarf galaxy, where $44 \%$ of the interloper stars have a velocity within 3- $\sigma$ of the systemic velocity of Fornax (35\% if $2.5-\sigma$ is used). In comparison, this is just $0.9 \%$ within $3-\sigma$ for Sextans.

In the third column of Fig. 4, we weed out contaminants according to the "line criterion", i.e. for which the closest synthetic spectrum in physical properties has a $\mathrm{EW}_{\mathrm{Mg}} / \Sigma \mathrm{W}_{\mathrm{CaT}}$ ratio that falls above the dividing line in Fig. 3. To derive the information on the line criterion, all interloper stars from the Besançon file were linked to the closest model within the grid of synthetic models (described in Sect. 2) in $[\mathrm{Fe} / \mathrm{H}], \log (g)$, and temperature space and the EWs of the CaT and Mg I lines of the closest model are used as their measurements. In all three galaxies it can clearly be seen that although a lot of contaminants are selected out (only $12-17 \%$ remains), the foreground consisting of giants remains (see also Fig. 5 for the distribution of contaminants in the $\mathrm{EW}_{\mathrm{Mg}}$ vs. $\Sigma \mathrm{W}_{\mathrm{CaT}}$ plane before and after velocity selection). The line criterion is less effective than the velocity criterion for Sextans and Sculptor. From Table 1, however, it is clear that for Fornax, which has a systemic velocity much closer to the one of MW thin disk with respect to the other two dSphs, the line criterion performs much better than the velocity criterion on its own.

The best results in all cases are obtained when the velocity and the line criterion are used in addition to each other, because 


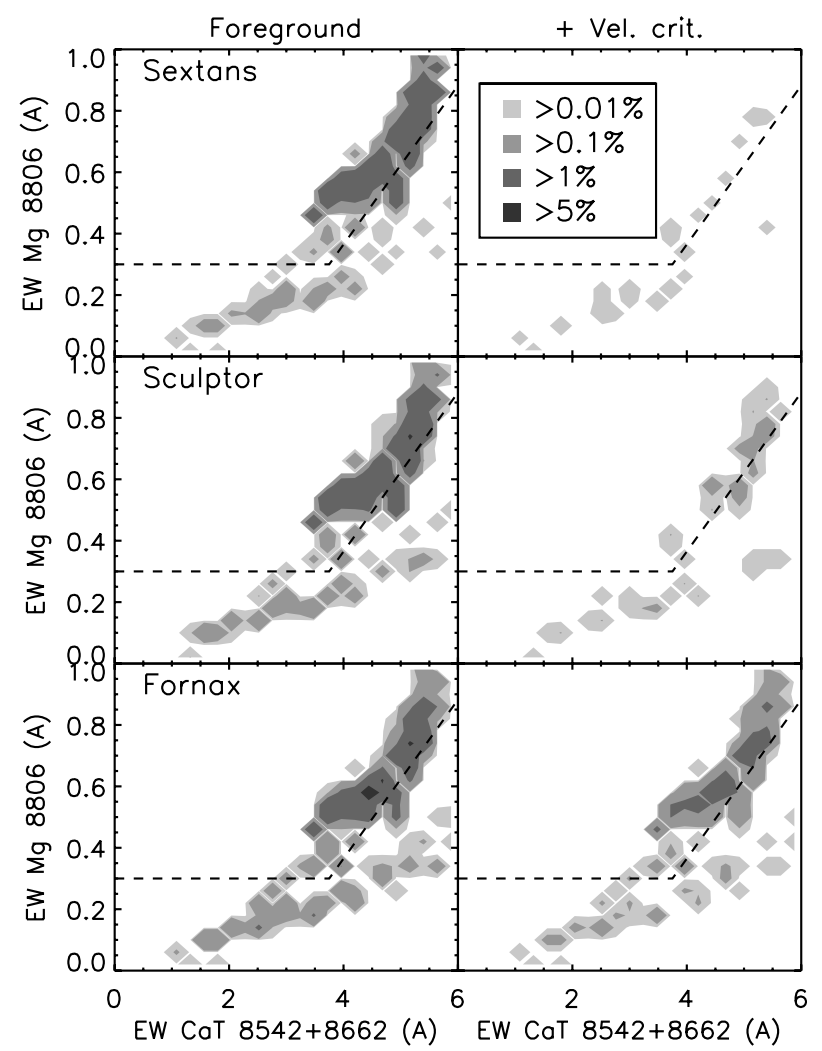

Fig. 5. Mg IEW and CaT $\Sigma W$ for the foreground stars drawn from the Besançon model towards the direction of the dwarf spheroidal galaxies Sextans (top row), Sculptor (middle row) and Fornax (bottom row). The left panels show all the foreground stars, and the right panels the distribution of foreground stars left after applying a velocity criterion of $3 \sigma$ around the systemic velocity of the galaxy. The dashed line indicates Eq. (1).

they are completely complementary in the sense that they will select out different sets of foreground stars. The line criterion will select especially on the physical properties (mostly gravity) of the star, and the velocity criterion just on its dynamics within the galaxy. Especially metal-rich dwarf foreground stars that have similar velocities and will be retained within the sample using a velocity cut will be very easily separated by the method using absorption lines. In the last column of Fig. 4 we show the result of a combination of both methods. In all galaxies a very large fraction of the interlopers can be weeded out and only a few percent of the interlopers still remain.

An additional value of this complementary use of both a velocity and line criterion is given in the last column of Table 1, where we relax the velocity criterion, allowing for a selection of member stars within a wider range of velocities, e.g. 4- $\sigma$, and find that this does not cause a large increase in the number of interlopers. This can prove extremely useful, since one of the concerns of imposing strict velocity criteria is the possible biases introduced by excluding member stars with more deviant velocities from the mean, for example when investigating the mass content of the galaxy from the velocity distribution of its stars.

\subsection{Predictions for the use of the method for other Milky Way dwarf galaxies, including the ultra-faints}

We now extend the previous analysis to the other early-type MW satellites. In the lower part of Table 1 (below the horizontal line) we summarize the relevant information for all those galaxies for which dynamical analyses have been carried out, including the UFDs (Strigari et al. 2008). For each of these galaxies, we extracted a catalog of foreground stars from the Besançon models and analyzed it in exactly the same way as described for the classical satellites Sextans, Sculptor, and Fornax. In the various galaxies, spectroscopic samples extend down to different depths, and, because of their sparsely populated RGB, most UFDs have also been surveyed in observations below the HB to increase the number of targets. For uniformity with the previous analysis, we consider only the region above the HB here, but this method will be effective at the metallicities expected for stars in UFDs also down to approximately one magnitude below the $\mathrm{HB}$, corresponding to the subgiant branch boxes in Fig. 3 (see also the Appendix for an exploration of the method performance to fainter magnitudes and bluer colors).

As seen in Table 1, in all galaxies the line criterion can weed out approximately $80-90 \%$ of the interlopers. The efficiency of a velocity criterion, on the other hand, is much more dependent on the particular galaxy and its systemic velocity. In most cases either the velocity criterion is the most effective or it performs comparably to the line criterion. However, for Canes Venatici I, Ursa Major I, and Willman 1, the line criterion outperforms a velocity criterion. The difference is very significant for the last two objects. For the three galaxies Hercules, Leo II, and Leo IV, adding an extra criterion that is gravity-dependent will also pay off significantly.

For all galaxies we studied, a combination of both criteria would lead to an excellent cleaning of the samples, leaving only up to a few percent of the foreground contamination. This is particularly important for the intrinsically faint small galaxies that have a sparsely populated RGB, where the samples of targets are bound to be small. In such cases, even if the number of contaminants is low, it can still represent a large fraction of the overall sample of observed stars. For example, we take as reference a faint system, such as UMa I, that has a luminosity in $V$ band of $1.4 \pm 0.4 \times 10^{4} L_{\odot}$ (Martin et al. 2008), and calculate what would be the expected number of RGB stars with respect to MW interlopers for such a system. Using the publicly available synthetic color-magnitude diagram code provided by the BaSTI team $^{1}$ and assuming that UMa I can be approximated by a $13 \mathrm{Gyr}$ old stellar population of $[\mathrm{Fe} / \mathrm{H}]=-2$ dex, about $20-25$ stars are produced in the region of the RGB above the HB (the numbers are from three different random realizations) for a system of $\sim 2 \times 10^{4} L_{\odot}$. For an exponential surface brightness profile, about $60 \%$ of the light is produced within one exponential radius, and therefore $60 \%$ of the number of stars are contained within one exponential radius, assuming no mass segregation. Using 6.7 arcmin as the exponential radius of UMa I (Martin et al. 2008), this would mean that approximately 12-15 RGB stars would be found over an area of $\sim 140 \mathrm{arcmin}^{2}$. Along the 1.o.s. to UMa I, the Besançon model gives approximately $40 \mathrm{MW}$ contaminants over such an area. Using the velocity criterion on its own would result into retaining about 10 interlopers in the sample, i.e. in comparable number to the RGB stars of the dwarf over the same area. Using both a velocity and line criterion, on the other hand, would only leave one or two contaminants in the sample.

Among the galaxies studied in this section, there are a few UFDs for which reaching about 2 mag down the HB appears to be particularly important for acquiring a statistically significant number of stars, such as for UMa I, UMa II, Leo IV, Com,

${ }^{1}$ http://albione.oa-teramo.inaf.it/. 


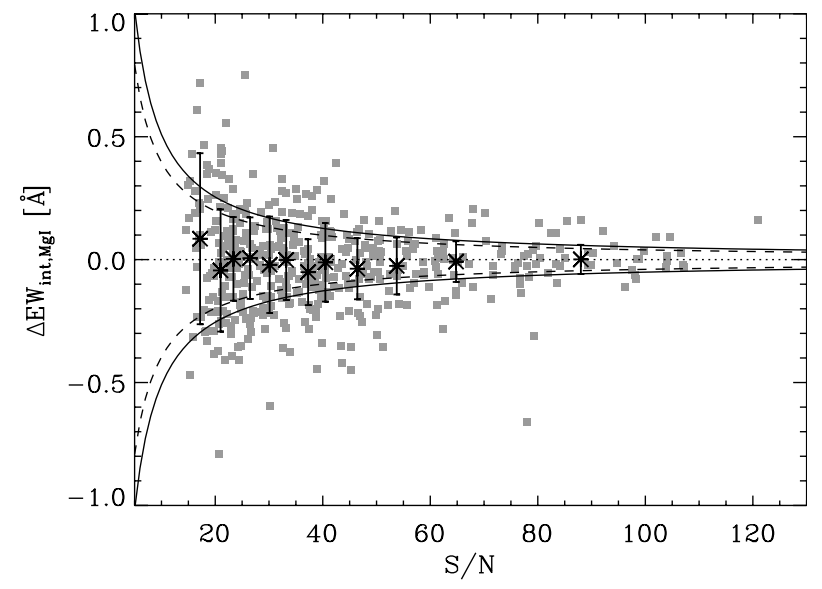

Fig. 6. Comparison between $\mathrm{Mg} I \mathrm{EW}$ measurements for stars with double measurements for the Sextans, Sculptor, and Fornax dSphs (59, 137, 289 stars, respectively). The figure shows the distribution of Mg IEW differences from integrated flux as a function of $\mathrm{S} / \mathrm{N}$ for the stars with $\mathrm{S} / \mathrm{N}$ per $\AA \geq 10$ and estimated error in velocity $\leq 5 \mathrm{~km} \mathrm{~s}^{-1}$ for each measurement. The solid lines indicate the $1-\sigma$ region for the error in the difference of EW; the dashed lines show the relation used in Battaglia et al. (2011). The asterisks indicate the median $\Delta \mathrm{EW}_{\mathrm{int}, \mathrm{MgI}}$ per $\mathrm{S} / \mathrm{N}$ bin, with the scaled m.a.d. shown as error bar.

CVn II, and Wil 1. We explore the performance of the method to fainter magnitudes in the Appendix. We restrict the analysis to the most compelling cases, UMa I, Leo IV, Wil 1, since for UMa II, Com, and CVn II the velocity criterion is already very effective on its own.

\section{The observed trend of Mg I vs. CaT EW}

We subsequently explore the observed trend of $\mathrm{Mg}$ IEW versus $\Sigma \mathrm{W}_{\mathrm{CaT}}$ in actual data and the way it compares to the predictions from the synthetic spectra, using the DART data-set for the three classical dSphs Sextans, Sculptor, and Fornax. In this way we are able to empirically explore the use of the $\mathrm{Mg}$ I and CaT lines EWs for these $\mathrm{dSphs}$ with different properties, i.e. located along different 1.o.s., with different systemic velocities and with stars covering different ranges of metallicities (see Table 1 for a summary). We used our intermediate resolution spectroscopic observations of these galaxies carried out at the VLT/FLAMES using the GIRAFFE spectrograph in Medusa mode with the LR8 grating, which covers the wavelength region 8206-9400 $\AA$ and therefore includes both the nIR CaT lines and the $\mathrm{Mg}$ I line at 8806.8 $\AA$. We refer the reader to the original papers for a general presentation of the dataset (Tolstoy et al. 2004; Battaglia et al. 2006, 2011; Helmi et al. 2006; Starkenburg et al. 2010).

The adopted data-reduction procedure is described in detail in Battaglia et al. (2008b). Here we briefly remind the reader that the EW of the $\mathrm{Mg}$ I line was derived by integrating the flux over $6 \AA$ around the central wavelength of the line as done for the synthetic spectra and in Battaglia et al. (2011). For this line, which is smaller than the CaT lines, this EW estimator provides less noisy EW measurements with respect to a Gaussian fit to the line.

The error in EW of the MgIline was derived by using the stars with double measurements (see Fig. 6). Assuming a similar $\mathrm{S} / \mathrm{N}$ for the individual measurements of each star, we obtain a typical error in the EW of the $\mathrm{Mg}$ I line, $\sigma_{\mathrm{EW}}, 0.06 \AA$ for Sextans, $0.13 \AA$ for Fornax, and $0.11 \AA$ for Sculptor as estimated from the scaled median absolute deviation from the median (m.a.d.) in the distribution of $\mathrm{MgIEW}$ differences. The weighted mean in the $\mathrm{Mg}$ I EW difference is consistent with zero in all cases $(0.01 \pm$ $0.02 \AA$ for Sextans, $-0.04 \pm 0.03 \AA$ for Fornax, and $-0.02 \pm$ $0.01 \AA$ for Sculptor). We derive the trend of $\sigma_{\mathrm{EW}}$ with $\mathrm{S} / \mathrm{N}$ by fitting the value of the scaled m.a.d. of the difference in $\mathrm{EW}_{\mathrm{Mg}}$ as a function of $\mathrm{S} / \mathrm{N}$ in bins containing at least 40 stars. Since the datasets for Sextans, Sculptor, and Fornax were obtained using the same instrument, observing strategy, and data reduction, we combine the data for the three galaxies. We obtain $\Delta \mathrm{EW}_{\mathrm{Mg}}=$ $\sqrt{2} \times \sigma_{\mathrm{EW}}=\sqrt{2} \times 3.6 /(\mathrm{S} / \mathrm{N})$, which would correspond to an error on the individual measurements of $\sigma_{\mathrm{EW}}=3.6 /(\mathrm{S} / \mathrm{N})$. Figure 6 shows how this relation differs from the one used in Battaglia et al. (2011), where only Sextans was analyzed.

In Fig. 7 we show the behavior of the MgIEW as a function of the l.o.s. velocity in the heliocentric system for the three dSphs. Here only spectra of higher quality (with $\mathrm{S} / \mathrm{N} / \AA>20$, velocity errors $<5 \mathrm{~km} \mathrm{~s}^{-1}$ and for which the difference between the $\mathrm{Mg}$ IEW estimated from the flux integration and the Gaussian fit is within five times the scaled m.a.d. of the distribution of $\left.\operatorname{MgI}\left|E W_{\text {int }}-E W_{\text {Gau }}\right|\right)$ are shown. This allows us to analyze the behavior of the $\mathrm{Mg}$ I line without being affected considerably by the noise in the measurements. In the figure we show the region of kinematic membership adopted in previous studies $( \pm 3 \sigma$ from the systemic velocity of the galaxy for Sculptor and Sextans, and $\pm 2.5 \sigma$ for Fornax $^{2}$ ). In previous works, stars within these velocity regions were considered as RGB stars that are probable members of the dSph.

As the figure shows, the majority of what are clearly contaminant stars (i.e. those stars with l.o.s. velocity well outside the region of kinematic membership) can be weeded out on the basis of a kinematic selection. However, from the figure it is also clear that the velocity distribution of contaminants extends in, and also beyond, the region of kinematic membership. Obviously, when applying a velocity criterion on its own, there will be contaminants with velocities consistent with membership, so classified as members. This is especially clear for the case of the Fornax $\mathrm{dSph}$, whose low systemic velocity falls well within the velocity distribution of MW foreground stars. Concerning the distribution in $\mathrm{Mg} \mathrm{IEW}$, it can be seen that in all three cases the majority of contaminants show a rather distinct distribution in $\mathrm{Mg}$ IEW with respect to the bulk of probable dSphs members, i.e. they mainly occupy the locus of $\mathrm{Mg}$ IEW $>0.5 \AA$. However, a few differences are present among the galaxies, both in the distribution of probable kinematic members and non-members in terms of their Mg I EW.

In the Sextans dSph $99 \%$ of the stars with velocities within the region of kinematic membership have $\mathrm{MgIEW}<0.5 \AA$, which says that it is very unlikely that RGB stars - genuine members - will be found with $\mathrm{Mg}$ IEW $>0.5 \AA$. Therefore a hard cut on the value of the Mg I EW as applied by B11 appears a good solution for weeding out as many contaminants as possible with 1.o.s. velocities consistent with kinematic membership, and at the same time retain in the sample the giants that are members of this dSph.

The same hard cut, though, cannot be applied unchanged to the Sculptor and Fornax dSphs. In the case of Sculptor, it is clear from Fig. 7 that a larger fraction of stars well outside the region of kinematic membership have $\mathrm{MgIEWs}<$ $0.5 \AA$ with respect to Sextans. This is because of the different

2 This stricter kinematic criterion was used in previous work to reduce the foreground contamination given the low systemic velocity of Fornax. 

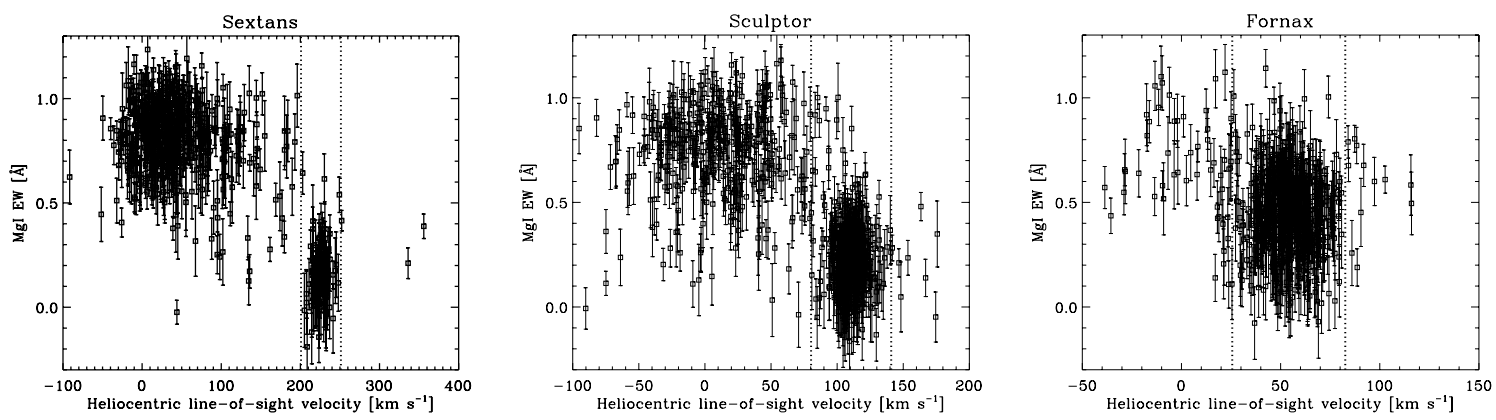

Fig. 7. Mg IEW versus 1.o.s. velocity in the heliocentric system (squares with error bars) for the Sextans, Sculptor, and Fornax dSphs (from left to right). The vertical dotted lines show the region of kinematic membership adopted in previous studies (i.e. with velocities within $\pm 3 \sigma$ from the systemic velocity of the $\mathrm{dSph}$ for Sextans and Sculptor, and $\pm 2.5 \sigma$ for Fornax).
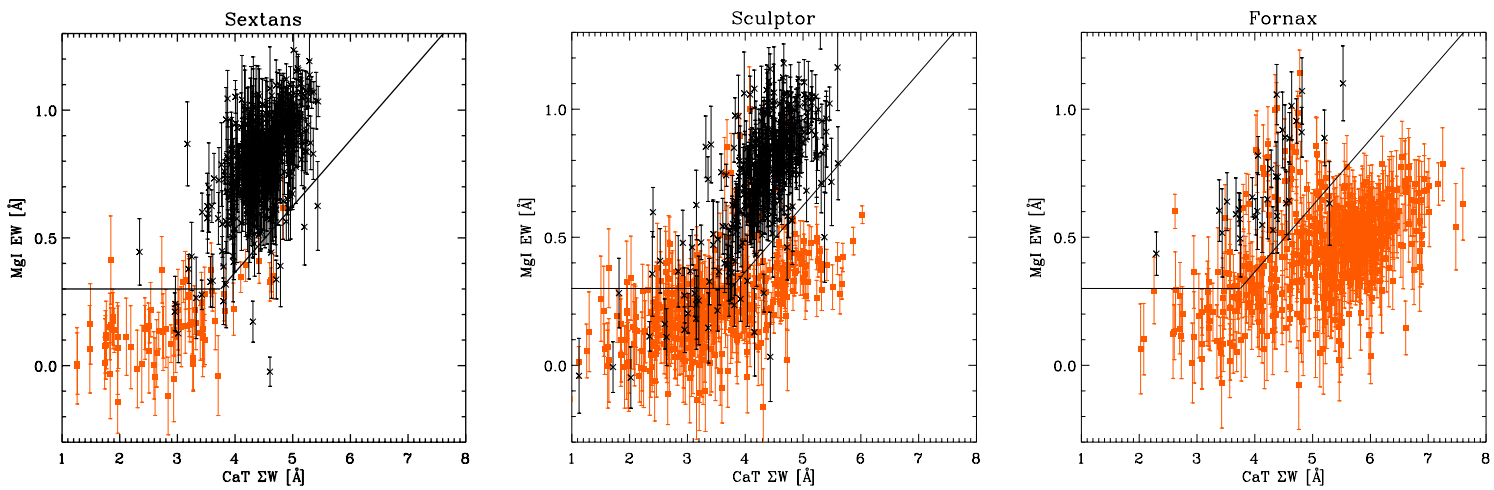

Fig. 8. Mg I EW versus $\Sigma W_{\mathrm{CaT}}$ for the Sextans, Sculptor and Fornax dSphs. The filled orange squares with error bars show the stars that are highly probable members (i.e. with velocities within $\pm 2 \sigma$ from the systemic velocity of the dSphs) and the black crosses the stars that are highly likely to be MW contaminants (i.e. with velocities at least $4 \sigma$ away from the systemic velocity of the dSphs). The solid line indicates the theoretical relation derived in Sect. 2.

mix of MW stars along the 1.o.s. to this galaxy (see Sect. 3 and Fig. 5). Furthermore, about $5 \%$ of kinematic members extend to MgIEW > $0.5 \AA$, so that blindly imposing the same cut-off as Sextans could result in discarding a considerable fraction of genuine members of the dSph. In the case of Fornax, all but one of the kinematic non-members have MgIEW > $0.5 \AA$, but also the kinematic members extend to high values of the Mg IEW, ranging from $\sim 0-1 \AA$. Therefore applying the same cut-off in Mg I EW would allow all contaminants to be removed, at the price of selecting out about $40 \%$ of the stars with velocities consistent with membership!

The reason for this is clearly seen in Fig. 8 where we plot the distribution in the CaT $\Sigma$ W- MgIEW plane for highly probable members of the dwarf and highly likely non-members. Since these are real data, and we do not know a priori what is a contaminant and what a genuine member of the dSph, to increase the probability of selecting members and non-members and make this distinction easier using only l.o.s. velocities, we plot the stars within 2- $\sigma$ of the systemic as members, and the stars beyond 4- $\sigma$ from the systemic as non-members. We emphasize that here we are interested in the behavior of the bulk of stars in both categories. For the likely members, a correlation is present between the $\Sigma \mathrm{W}_{\mathrm{CaT}}$ and the MgIEW, so that stars with larger $\Sigma \mathrm{W}_{\mathrm{CaT}}$ display larger MgIEW. At $\Sigma \mathrm{W}_{\mathrm{CaT}} \gtrsim 6 \AA$, the typical $\mathrm{Mg}$ IEW is $>0.5 \AA$, and in Fornax, which is more metal-rich than Sextans and Sculptor, most of the stars in our dataset occupy the region $\Sigma \mathrm{W}_{\mathrm{CaT}} \gtrsim 6 \AA$. The correlation in MgIEW vs. $\Sigma \mathrm{W}_{\mathrm{CaT}}$ observed in the data is consistent with the results from the analysis using synthetic spectra (Sect. 2) and appears similar for the different dSphs at similar values of $\Sigma \mathrm{W}$. This confirms the result of Sect. 2 for which a general criterion can be applied in MgIEW vs. $\Sigma \mathrm{W}_{\mathrm{CaT}}$ to separate contaminants and probable members of the dSphs. Obviously in the case of observations the errors in the measurement of the EWs of the lines also need to be taken into consideration when comparing the location of the star in the Mg I vs. $\Sigma W_{\text {CaT }}$ plane with the line separating dwarfs and giants derived from the models.

\subsection{Applying the line criterion to data}

In the previous sections we have proven the applicability and validity of the method; it is therefore interesting to see how the application of the line criterion to available datasets, e.g. DART, would perform. Here, when referring to "contaminants" we refer to stars classified as such on the basis of the line criterion.

As visual inspection of Fig. 8 already suggests, a significant number of stars that have l.o.s. velocities consistent with being highly probably members of the Fornax dSphs are actually found above the dividing line between dwarf and giant stars derived in Sect. 2, and therefore are most likely MW interlopers that were misclassified because they went unrecognized even by a strict velocity selection. Fewer such objects are also present in Sextans and Sculptor.

We apply the method to those stars that pass our usual selection criteria (i.e. $\mathrm{S} / \mathrm{N}>10 / \AA$, velocity errors $<5 \mathrm{~km} \mathrm{~s}^{-1}$ and $\left.\mathrm{CaT}\left|\mathrm{EW}_{\text {int }}-\mathrm{EW}_{\text {gau }}\right|<2 \AA\right)^{3}$. Because of the measurement errors, the distribution of highly probable members on this plane

\footnotetext{
${ }^{3}$ Since part of the targets for Sculptor were chosen from preliminary photometry, a handful of them turned out to have colors and magnitudes well outside the overall selection box for RGB stars from the definitive photometry. We exclude these objects by restricting ourselves to $V-I>$ 0.5 and $V>16.5$.
} 
is inflated and in some cases goes over the separation line between dwarf and giant stars. In this work we account for the errors in Mg IEW in a simple way, i.e. we consider as "giants" those stars with $\mathrm{EW}_{\mathrm{Mg} I}$ lower or within $1 \sigma_{\mathrm{EW}}$ from the $\mathrm{Mg}$ I EW obtained by applying Eq. (1) (see dividing line in Fig. 3). We define as "contaminants" those stars with values of Mg IEW more than $1 \sigma_{\mathrm{EW}}$ higher than the value obtained by applying Eq. (1). A more detailed approach, which would for example consider the combined probability of membership from the velocity and $\mathrm{Mg}$ I EW distribution in a statistical way, is beyond the scope of this paper, but we refer the reader to Walker et al. (2009b) and Martinez et al. (2011) for works dealing with the issue of membership with comprehensive statistical analyses.

The Sextans dSph was previously analyzed, so here we just mention that, by considering the error in $\mathrm{Mg}$ I line in the selection, only three stars are considered as contaminants, instead of the six stars rejected in B11. This has no influence on the results other than bringing back into the sample stars that had discrepant metallicities with respect to the body of the distribution, indicating that perhaps this selection may be too generous. The rejected stars account for less than $2 \%$ of the overall sample. This may seem surprising since Sextans is a very low surfacebrightness object, with a CMD heavily contaminated by MW stars, and therefore one may expect a large amount of contaminants. However, the systemic velocity of the object, far from the one of the MW disks, appears to allow for a good cleaning of the sample only on the basis of the l.o.s. velocity.

Among the stars within the region of kinematic membership, Fornax and Sculptor both have a percentage of $~ 5 \%$ of stars classified as contaminants according to the line criterion. For Fornax, kinematic members were chosen from a $2.5 \sigma$ cut in our previous works. If we consider the kinematic membership region as the velocity range between $\pm 3 \sigma$ from the systemic velocity, the fraction of interlopers increases to $6.5 \%$ of the total. These contaminant stars are found at all projected radii, but their relative contribution to the number of members becomes increasingly significant towards the outer parts, accounting for example for about $45 \%$ and $35 \%$ of the kinematic members at $R>1.0 \mathrm{deg}$ for Fornax and Sculptor, respectively. For comparison, between 0.5 and $1.0 \mathrm{deg}(\sim 0.4$ and 0.8 nominal tidal radii, according to the value from Irwin \& Hatzidimitriou 1995), the fraction drops to $6.3 \%$ in Sculptor. The fraction of contaminants found for these two objects are very similar to each other, even though Table 1 would have suggested that a much larger fraction would be found for Fornax. This is most likely due to a combination of factors such as the different area covered and the much larger surface brightness of Fornax with respect of Sculptor, which permits us to pick out a larger number of member stars from the foreground.

Figure 9 (top) shows the distribution of the analyzed stars for Sculptor and Fornax on the $\mathrm{CaT}[\mathrm{Fe} / \mathrm{H}]$ versus heliocentric l.o.s. velocity plane. The combination of these quantities can already provide information on the probability of membership of a star to the MW or to the dwarf galaxy, since on this plane the great majority of those stars that are clearly unbound to the dwarf galaxy (MW stars) have a different distribution than the velocity members (likely RGB stars of the dSphs). For example, it is clear that the region with $-2<[\mathrm{Fe} / \mathrm{H}]<-1$ is expected to be the most contaminated one. The figure also shows that most of the stars excluded by the line criterion alone indeed have CaT $[\mathrm{Fe} / \mathrm{H}]$ values in the range where most contaminants were expected, highlighting that the method presented here, which makes no a priori selection in metallicity and velocity, is indeed able to find interlopers.
We note that the $\mathrm{CaT}[\mathrm{Fe} / \mathrm{H}]$ relation is empirically calibrated for RGB stars (above the HB) and relies on the assumption that all the stars can be considered at the same distance. While this is a reasonable assumption for the stars belonging to the dSphs analyzed here, this is not the case for the bulk of MW interlopers, which are located over a range of distances - mostly within a few kpc from the Sun - and have markedly different gravities than RGB stars. Therefore, the $\mathrm{CaT}[\mathrm{Fe} / \mathrm{H}]$ values for the stars that are probable non-members are not necessarily indicative of their true metallicity.

Figure 9 (top) also illustrates what would be the result of relaxing the kinematic criterion to $4 \sigma$ for both galaxies ${ }^{4}$ : the fraction of interlopers becomes about $8 \%$ for Fornax and $6.5 \%$ for Sculptor, and the new contaminants are found at lower velocities than the systemic, towards the velocities of the disk, as would be expected. The bottom panels of Fig. 9 show the metallicity distributions as a function of projected radius resulting from applying the line criterion in addition to previous kinematic criteria, so the conclusions from our previous works in terms of changes of metallicity with radius holds. Also the smaller velocity dispersion of metal-rich stars than metal-poor stars is still present. For Sculptor it can be seen that several of the stars with $[\mathrm{Fe} / \mathrm{H}]>-1.7$ dex at $R>0.6$ deg may actually not belong to Sculptor, making the variation in metallicity properties with projected radius even clearer.

One can therefore conclude that the main properties of systems for which samples of several hundred individual stars are available, such as the majority of classical dSphs, are robust. It is also clear that methods of the kind proposed here can potentially be very helpful for those analyses whose conclusions rely heavily on the properties of stars in the outer parts of these systems, where the ratio of unrecognized interlopers to member stars increases, as well for studies of several of the fainter dwarf galaxies such as the UFDs.

\section{Discussion and conclusions}

In this work we have addressed the problem of identifying MW stars in samples of kinematically selected members stars in Local Group galaxies. The presence of such interlopers may contaminate the derived properties of the target galaxy and this is a general issue in studies of resolved stellar populations of Local Group galaxies.

The method that we propose is mainly meant for spectroscopic samples in the CaT region whose targets are selected from broad band photometry to lie in the region of the CMD that covers the RGB of the target galaxy (above or approximately one magnitude below the HB). This is the case for the large majority of data for "classical" dSphs and more distant Local Group dwarf galaxies; for several UFDs a significant fraction of the targets lie in that magnitude range. We explored the combined use of the MgIEW at $8806.8 \AA$ and the nIR CaT $\Sigma \mathrm{W}$ as a way of distinguishing between contaminants and members on the basis of physical characteristics of the star, mainly gravity, since the targeted stars are RGB stars, while the MW interlopers will mostly consist of dwarf stars.

For this we used synthetic spectra over a range of metallicity, temperature, and gravity covering the expected age and metallicity range of stars in Local Group dwarf galaxies. We found that a relation between the $\mathrm{MgIEW}$ and the CaT $\Sigma \mathrm{W}$ can be applied to distinguish between dwarf and giant stars above the HB for metallicities $[\mathrm{Fe} / \mathrm{H}]>-2$ dex and for (sub)giant stars below the

\footnotetext{
${ }^{4}$ Here we use the velocity dispersion values from Table 1.
} 

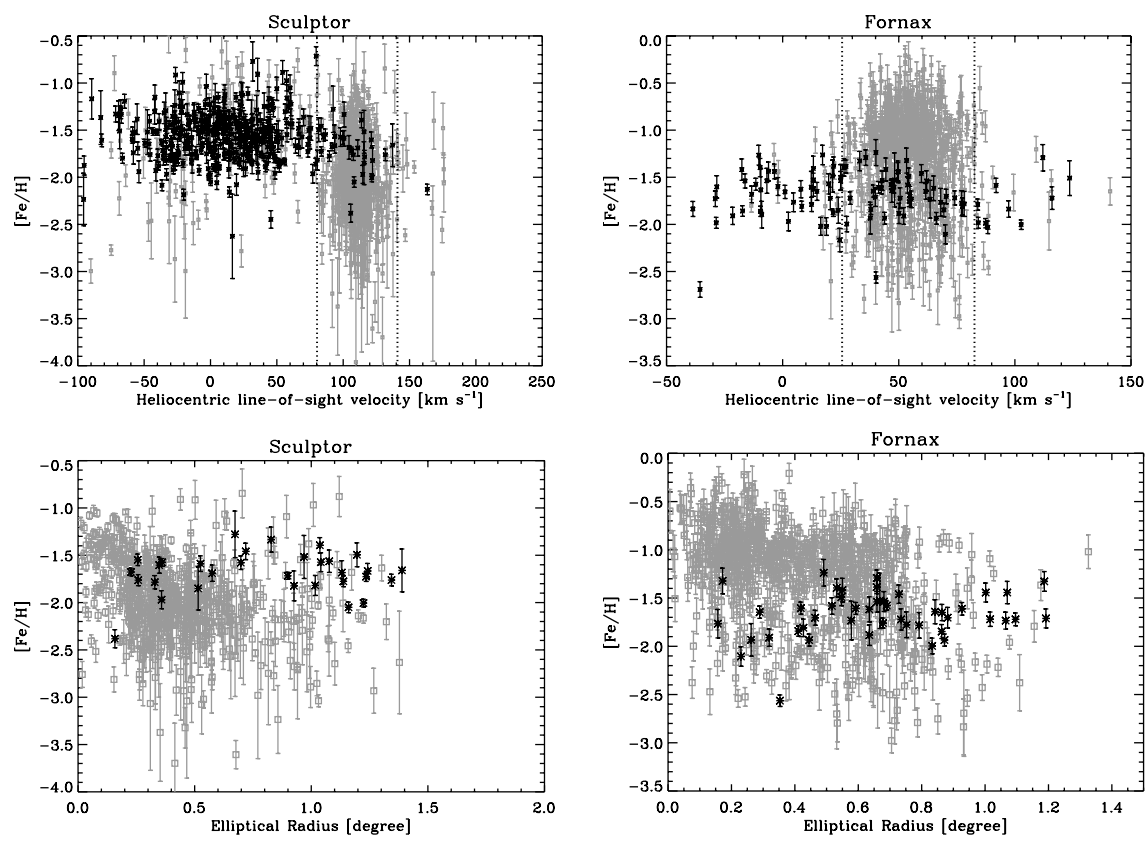

Fig. 9. Application of the line method to the Sculptor (left) and Fornax (right) dSphs, using the VLT/FLAMES DART dataset. Top panels: distribution on the CaT $[\mathrm{Fe} / \mathrm{H}]$ versus 1.o.s. heliocentric velocity for the stars with $\mathrm{S} / \mathrm{N} / \AA \AA^{\circ}>10$, error in velocity $<5 \mathrm{~km} \mathrm{~s}^{-1}$, and $\mathrm{CaT}\left|\mathrm{EW}_{\text {int }}-\mathrm{EW}_{\text {gau }}\right|<2 \AA$. The black squares with error bars show those stars whose $\mathrm{Mg}$ I EW was either lower than or within $1 \sigma_{\mathrm{EW}}$ from the Mg I EW given by Eq. (1), while gray squares with error bars show those stars whose $\mathrm{Mg}$ IEW was more than $1 \sigma_{\mathrm{EW}}$ larger than the Mg IEW given by Eq. (1). The vertical lines indicate the region of kinematic membership from our previous works. Bottom panel: $\mathrm{CaT}[\mathrm{Fe} / \mathrm{H}]$ versus elliptical radius for kinematic members (black: with MgIEW within $1 \sigma_{\mathrm{EW}}$ from Eq. (1); gray: with $\mathrm{Mg} I \mathrm{EW}$ more than $1 \sigma_{\mathrm{EW}}$ away from Eq. (1)).

$\mathrm{HB}$ for $-2 \leq[\mathrm{Fe} / \mathrm{H}] \leq-1$. Since at lower metallicities the main contribution of contaminants comes from the MW stellar halo, which is the least dominant component in terms of stellar mass (and therefore number of stars), only a negligible amount of interlopers is expected below this metallicity.

The relation between the $\mathrm{MgIEW}$ and the CaT $\Sigma \mathrm{W}$ derived from analyzing synthetic spectra applies well to the trend observed in actual data for a sample of three classical dSphs (Sextans, Fornax, and Sculptor), whose stars cover different ranges of age and metallicity. The 1.o.s. to the target object and the velocity of the center of mass of the galaxy (the systemic velocity) are important parameters because they determine the amount and mix of contaminants that will be present in the kinematically selected samples of stars. This means that there will be objects for which the l.o.s. velocity selection is already very efficient, while others that will greatly benefit from an additional criterion like the one explored here. We find that the addition of this method to the information from the l.o.s. velocity can be particularly beneficial, for example, for Canes Venatici I, Leo II, Fornax, Hercules, Ursa Major I, and Willman 1. For the last four galaxies, the use of the Mg IEW method on its own is considerably more efficient than the selection in l.o.s. velocity.

It should be noted that the contamination by giant stars from the MW is not removed by this method, the proposed relation will retain as many giant stars as possible and throw away only dwarf contaminants. Another very important parameter in determining the ratio between contaminants and stars member to the dwarf is the ratio between the projected number density of MW and dwarf galaxy stars. Besides varying from object to object, this ratio changes within the object itself simply because of the declining surface density of stars in dwarf galaxies, so that weeding out interlopers will become particularly important in the outer parts of the systems, and for intrinsically faint objects.

This method has the advantage of being very easily implemented, since it relies on measuring EWs for relatively large lines such as the nIR CaT and MgI. These lines are close in wavelength, so that several existing studies of MW dSphs covering this wavelength region could benefit from this extra information.

Acknowledgements. The authors would like to thank Mike Irwin for useful conversations that have inspired this work, comments and for the use of his equivalent width extraction routine. We thank Eline Tolstoy, Amina Helmi and Vanessa Hill for useful comments. We also kindly acknowledge Bertrand Plez for making his linelists and Turbospectrum code available. E.S. gratefully acknowledges support through an NWO-VICI grant. We acknowledge the International Space Science Institute (ISSI) at Bern for their funding of the team "Defining the full life-cycle of dwarf galaxy evolution: the Local Universe as a template". The research leading to these results has received funding from the European Union Seventh Framework Program (FP7/2007-2013) under grant agreement number PIEF-GA-2010-274151. This work has made use of BaSTI web tools. The authors would like to thank the BaSTI team for making publicly available the synthetic color-magnitude diagram web tool. G.B. is grateful to the Kapteyn Astronomical Institute for hospitality and financial support during part of this work.

\section{Appendix A}

Here we explore how the line criterion would perform down to 2 mag below the HB for Uma I, Wil 1, and Leo IV, i.e. those UFDs for which the application of the line criterion would considerably improve the elimination of foreground stars, but whose faintness makes it very difficult to acquire several targets, even reaching down 1 mag below the $\mathrm{HB}$. We do so by increasing the temperature range and including models as hot as $5500 \mathrm{~K}$. While this results in colors that are only milder bluer than previously considered, i.e. $V-I \sim 0.8$, it allows the magnitude range of the sub-giant branch boxes to be extended about 1 mag at the fainter limit.

Figure A.1 shows that qualitatively the results of Sect. 2 still hold. At $[\mathrm{Fe} / \mathrm{H}]=-2$ and -1 it is possible to make a distinction between dwarfs and giants/subgiant stars. At $[\mathrm{Fe} / \mathrm{H}]=0$ 

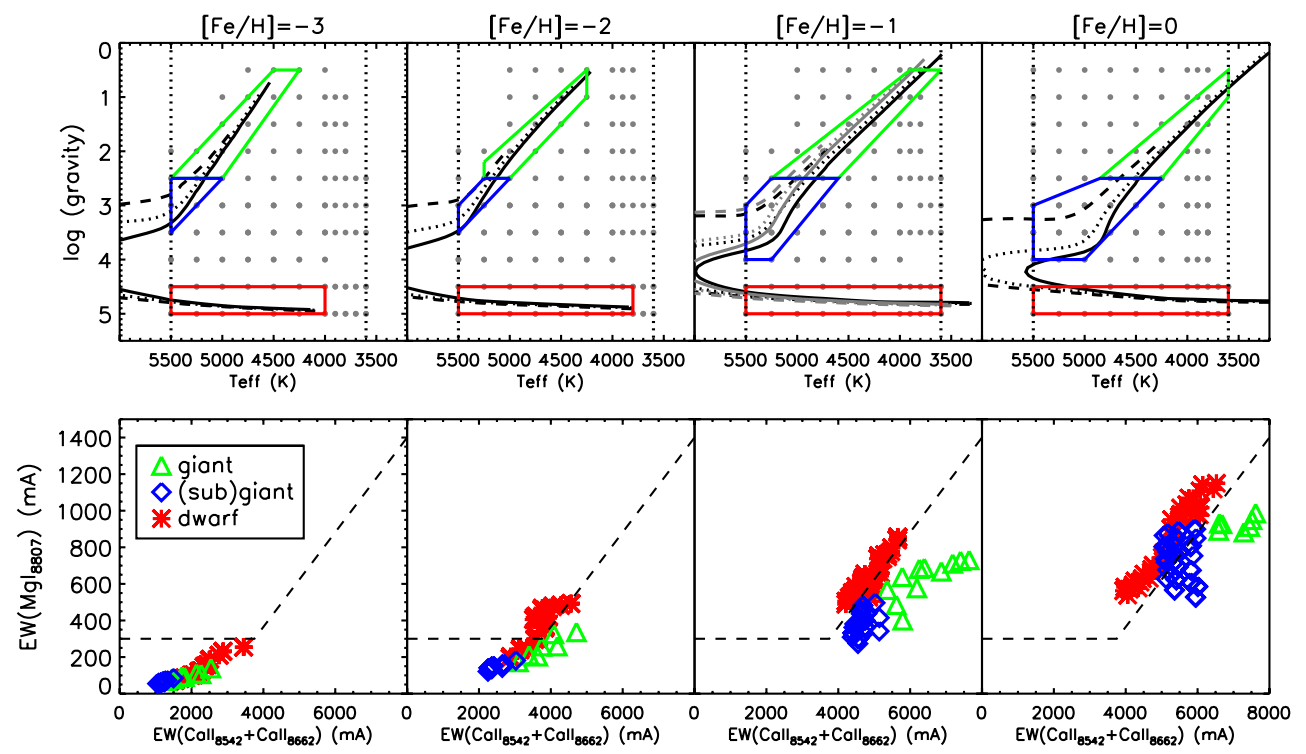

Fig. A.1. As in Fig. 3 but imposing an upper limit of $5500 \mathrm{~K}$ for the selection in temperature, rather than $5250 \mathrm{~K}$.

Table A.1. Properties of the foreground contamination towards Leo IV, UMa I and Wil 1.

\begin{tabular}{lcccc}
\hline \hline Galaxy & $\begin{array}{c}N_{\text {cont }} \\
/ 4 \mathrm{deg}^{2}\end{array}$ & $\begin{array}{c}\%\left(N_{\text {cont }}\right) \\
\text { vel. crit. }(3 \sigma)\end{array}$ & $\begin{array}{c}\%\left(N_{\text {cont }}\right) \\
\text { line crit. }\end{array}$ & $\begin{array}{c}\%\left(N_{\text {cont }}\right) \\
\text { both }(3 \sigma)\end{array}$ \\
\hline Leo IV & 8063 & $13 \%$ & $25 \%$ & $5 \%$ \\
Ursa Major I & 7147 & $34 \%$ & $17 \%$ & $5 \%$ \\
Willman 1 & 3806 & $36 \%$ & $15 \%$ & $3 \%$ \\
\hline
\end{tabular}

Notes. For the definition of the columns see Table 1.

subgiants overlap with both the locus of dwarfs and giants, but dwarfs and giants remain distinct. As before, at $[\mathrm{Fe} / \mathrm{H}]=-3$ the various types of stars overlap in the $\Sigma \mathrm{W}_{\mathrm{CaT}}-\mathrm{EW}_{\mathrm{Mg}}$ plane.

Obviously the actual efficiency in removing contaminants will change from what is considered in Sect. 3.2, because by increasing the temperature range at the higher temperature end, we not only increase the number of contaminants but also include hotter MW stars. Table A.1 shows the results when selecting stars from the Besançon model to have $0.8<V-I<2.1$ and magnitude from the tip of the RGB (as in Sect. 3) down to 2 mag below the HB. The fraction of contaminant stars retained in the sample by both velocity and line criterion increases, but the combined efficiency is still very good, with only $3-5 \%$ of interlopers retained. It should be also noted that the line criterion still outperforms the velocity criterion for Ursa Major I and Willman 1.

\section{References}

Adén, D., Feltzing, S., Koch, A., et al. 2009a, A\&A, 506, 1147 Adén, D., Wilkinson, M. I., Read, J. I., et al. 2009b, ApJ, 706, L150 Alvarez, R., \& Plez, B. 1998, A\&A, 330, 1109 Barklem, P. S., Christlieb, N., Beers, T. C., et al. 2005, A\&A, 439, 129 Battaglia, G., Tolstoy, E., Helmi, A., et al. 2006, A\&A, 459, 423 Battaglia, G., Helmi, A., Tolstoy, E., et al. 2008a, ApJ, 681, L13 Battaglia, G., Irwin, M., Tolstoy, E., et al. 2008b, MNRAS, 383, 183 Battaglia, G., Tolstoy, E., Helmi, A., et al. 2011, MNRAS, 411, 1013 Belokurov, V., Zucker, D. B., Evans, N. W., et al. 2007, ApJ, 654, 897
Catelan, M., \& Cortés, C. 2008, ApJ, 676, L135

Coleman, M. G., Da Costa, G. S., Bland-Hawthorn, J., \& Freeman, K. C. 2005, AJ, 129, 1443

Demarque, P., Woo, J., Kim, Y., \& Yi, S. K. 2004, ApJS, 155, 667

Faria, D., Feltzing, S., Lundström, I., et al. 2007, A\&A, 465, 357

Gilbert, K. M., Guhathakurta, P., Kalirai, J. S., et al. 2006, ApJ, 652, 1188

Gilmore, G., Wilkinson, M. I., Wyse, R. F. G., et al. 2007, ApJ, 663, 948

Gustafsson, B., Edvardsson, B., Eriksson, K., et al. 2008, A\&A, 486, 951

Helmi, A., Irwin, M. J., Tolstoy, E., et al. 2006, ApJ, 651, L121

Irwin, M., \& Hatzidimitriou, D. 1995, MNRAS, 277, 1354

Kaiser, N., Aussel, H., Burke, B. E., et al. 2002, in SPIE Conf. Ser. 4836, ed. J. A. Tyson, \& S. Wolff, 154

Keller, S. C., Schmidt, B. P., Bessell, M. S., et al. 2007, PASA, 24, 1

Kirby, E. N., Guhathakurta, P., Simon, J. D., et al. 2010, ApJS, 191, 352

Koch, A., Grebel, E. K., Wyse, R. F. G., et al. 2006, AJ, 131, 895

Koch, A., Grebel, E. K., Gilmore, G. F., et al. 2008, AJ, 135, 1580

Letarte, B., Hill, V., Tolstoy, E., et al. 2010, A\&A, 523, A17

Martin, N. F., Ibata, R. A., Chapman, S. C., Irwin, M., \& Lewis, G. F. 2007, MNRAS, 380, 281

Martin, N. F., de Jong, J. T. A., \& Rix, H.-W. 2008, ApJ, 684, 1075

Martinez, G. D., Minor, Q. E., Bullock, J., et al. 2011, ApJ, 738, 55

Mateo, M. L. 1998, ARA\&A, 36, 435

Morrison, H. L., Olszewski, E. W., Mateo, M., et al. 2001, AJ, 121, 283

Muñoz, R. R., Majewski, S. R., Zaggia, S., et al. 2006, ApJ, 649, 201

Plez, B. 2008, Phys. Scr. T, 133, 014003

Ramírez, I., \& Meléndez, J. 2005, ApJ, 626, 465

Rich, R. M., Corsi, C. E., Cacciari, C., et al. 2005, AJ, 129, 2670

Robin, A. C., Reylé, C., Derrière, S., \& Picaud, S. 2003, A\&A, 409, 523

Schiavon, R. P., Barbuy, B., Rossi, S. C. F., \& Milone, A. 1997, ApJ, 479, 902

Simon, J. D., \& Geha, M. 2007, ApJ, 670, 313

Simon, J. D., Geha, M., Minor, Q. E., et al. 2011, ApJ, 733, 46

Spinrad, H., \& Taylor, B. J. 1971, ApJS, 22, 445

Starkenburg, E., Hill, V., Tolstoy, E., et al. 2010, A\&A, 513, A34

Strigari, L. E., Bullock, J. S., Kaplinghat, M., et al. 2008, Nature, 454, 1096

Tolstoy, E., Irwin, M. J., Helmi, A., et al. 2004, ApJ, 617, L119

Tolstoy, E., Hill, V., Irwin, M., et al. 2006, The Messenger, 123, 33

Tolstoy, E., Hill, V., \& Tosi, M. 2009, ARA\&A, 47, 371

Venn, K. A., Irwin, M., Shetrone, M. D., et al. 2004, AJ, 128, 1177

Walker, M. G., Mateo, M., Olszewski, E. W., et al. 2006, ApJ, 642, L41

Walker, M. G., Mateo, M., Olszewski, E. W., et al. 2009a, ApJ, 704, 1274

Walker, M. G., Mateo, M., Olszewski, E. W., Sen, B., \& Woodroofe, M. 2009b, AJ, 137, 3109

Yi, S., Demarque, P., Kim, Y.-C., et al. 2001, ApJS, 136, 417

Zucker, D. B., Belokurov, V., Evans, N. W., et al. 2006a, ApJ, 650, L41

Zucker, D. B., Belokurov, V., Evans, N. W., et al. 2006b, ApJ, 643, L103 